\title{
Inertial particle segregation by turbulence
}

\author{
J. C. H. Fung \\ Department of Mathematics, The Hong Kong University of Science and Technology, Clear Water Bay, Kowloon, Hong Kong \\ J. C. Vassilicos \\ Turbulence and Mixing Group, Department of Aeronautics, Imperial College, Prince Consort Road, \\ South Kensington, London SW7 2BY, United Kingdom \\ (Received 3 December 2002; published 28 October 2003)
}

\begin{abstract}
We study collections of heavy and light small spherical particles initially well mixed with each other, subjected to linear (Stokes) drag force and gravity, and falling through a fluid turbulence. We introduce the segregation power spectrum, which we use to define the segregation length scale. Kinematic simulation predicts that the turbulence can segregate heavy and light falling particles and leads to a well-defined segregation length scale. The properties of this length scale and of the segregation power spectrum used to define it are discussed and, where possible, explained.
\end{abstract}

DOI: 10.1103/PhysRevE.68.046309

PACS number(s): 47.27.Eq, 47.27.Qb, 47.27.Gs

\section{INTRODUCTION}

Suspended particles such as soil dust from wind erosion and man-made pollutants can be found in many turbulent flows. Recent experimental and computational studies of the motion of small particles in turbulent flows have shown how the displacement $\mathbf{x}_{p}$ and velocity $\mathbf{v}(t)$ of a particle differ from those $\left[\mathbf{x}_{f}\right.$ and $\left.\mathbf{u}(t)\right]$ of a fluid element, depending on the size of the particle and on the drag and body forces acting on it. Maxey [1], Squires and Eaton [2], Wang and Maxey [3], and Fung [4] showed that the distribution of particles in a turbulent flow is not uniform; instead, particles tend to cluster preferentially in regions of low vorticity and high strain rate (see also the comprehensive recent study by Ahmed and Elghobashi [5] and references therein). This means that models of particle dispersion in turbulent flows should incorporate some of the spatiotemporal structural features of turbulent flow field realizations. But this also means that turbulent flows may be able to segregate particles of different sizes or densities so that initially well-mixed collections of different types of particle should become unmixed by the turbulence and give rise to pockets with predominantly one type of particle. Is it possible to define a length scale characterizing this segregation?

In this paper we investigate the ability of the turbulence to segregate particles of different inertias (i.e., sizes or densities) falling through turbulence and propose a definition for the segregation length scale. We use kinematic simulation (KS), which incorporates some degree of spatiotemporal flow structure designed to mimic some of the salient features of small-scale turbulence. For this paper's purpose of qualitative demonstration, we concentrate attention on twocomponent homogeneous and isotropic turbulence, and the KS used is designed to have a Kolmogorov $-5 / 3$ energy spectrum and corresponding frequencies in its temporal structure.

The paper is organized as follows. In Sec. II we introduce the equation of motion for heavy particles. To be solved, this equation requires modeling of the turbulence, and in Sec. III we describe KS. The results of numerical simulations con- cerning mixtures of heavy particles with different inertias in a turbulent flow are presented in Sec. IV, and we conclude in Sec. V.

\section{EQUATION OF MOTION FOR INERTIAL PARTICLES}

We consider a particle of mass $m_{p}$ and density $\rho_{p}$, located at a point $\mathbf{x}_{p}(t)$ and moving with velocity $\mathbf{v}(t)$ in a fluid of density $\rho_{f}$ and kinematic viscosity $\nu$. The fluid velocity field is given by $\mathbf{u}(\mathbf{x}, t)$, and we denote the instantaneous fluid velocity at the position of the particle by $\mathbf{u}\left(\mathbf{x}_{p}, t\right)$. Then the equation of motion for the particle is

$$
m_{p} \frac{d \mathbf{v}}{d t}=\mathbf{F}(\mathbf{u}, \mathbf{v}, t)
$$

The force $\mathbf{F}$ on the particle, which is still the subject of much current research, is made up of many different contributions, including, for example, the acceleration force $\mathbf{F}_{A}$, the lift force $\mathbf{F}_{L}$, the body force $\mathbf{F}_{B}$, the drag force $\mathbf{F}_{D}$, and the Boussinesq-Basset (often just called Basset-see Vojir and Michaelides [6]) history term (see Hunt et al. [7] and Mei [8] for reviews).

If a particle in a turbulent flow is heavy (i.e., $\rho_{p} \gg \rho_{f}$ ) and spherical, and if its radius $a$ is small relative to the smallest length scale of the turbulence (the Kolmogorov length scale $\eta$, which is about $1 \mathrm{~mm}$ in the lower atmosphere), then only the drag and buoyancy forces are important (terms involving the pressure gradient force, the virtual mass force, and the Basset force can be neglected), and Eq. (1) becomes

$$
m_{p} \frac{d \mathbf{v}}{d t}=\mathbf{F}_{D}+\mathbf{F}_{B}
$$

where $\mathbf{F}_{B}=m_{p} \mathbf{g}$. $\mathbf{g}$ is the acceleration due to gravity and it is oriented downward parallel to the $y$ axis, which points upward. In the limit where the particle Reynolds number $\operatorname{Re}_{p}=2 a\left|\mathbf{u}\left(\mathbf{x}_{p}, t\right)-\mathbf{v}(t)\right| / \nu$ is much smaller than 1 , the drag force can be approximated by the Stokes linear form $\mathbf{F}_{D}$ $=6 \pi a \mu\left[\mathbf{u}\left(\mathbf{x}_{p}, t\right)-\mathbf{v}(t)\right]$, and Eq. (2) becomes 


$$
\frac{d \mathbf{v}}{d t}=\frac{1}{\tau_{p}}\left[\mathbf{u}\left(\mathbf{x}_{p}, t\right)-\mathbf{v}(t)\right]+\mathbf{g},
$$

where the relaxation time $\tau_{p}$ is given by

$$
\tau_{p}=\frac{m_{p}}{6 \pi a \rho_{f} \nu}=\frac{2 \rho_{p} a^{2}}{9 \rho_{f} \nu} .
$$

A Stokes drag is assumed here. Previous experience indicates that the Stokes drag gives qualitatively similar results as some nonlinear empirical drag laws (Reeks [9] Wang and Stock [10], Wang and Maxey [3], and Fung [11]). Despite the restrictions imposed, Eq. (3) is applicable to many different problems, such as aerosols in gases and small particles in water. Under most atmospheric conditions, Eq. (3) would apply, for instance, to aerosol particles or cloud drops with radii less than about $100 \mu \mathrm{m}$. To be solved, Eq. (3a) must of course be coupled to

$$
\frac{d \mathbf{x}_{p}}{d t}=\mathbf{v}(t)
$$

If we set $\mathbf{u}=\mathbf{0}$ in Eq. (3a) and take the limit $t \rightarrow \infty$, we obtain $\mathbf{v}(t)=\mathbf{V}_{T}$ where $\mathbf{V}_{T}=\tau_{p} \mathbf{g}$ is the Stokes settling velocity (terminal velocity in a still fluid). When the velocity field is turbulent, however, $\mathbf{u}$ needs to be modeled before Eq. (3) can be solved. In KS, the turbulent velocity field $\mathbf{u}(\mathbf{x}, t)$ is modeled as a sum of random incompressible Fourier modes with a $-5 / 3$ energy spectrum. We assume that the particles do not significantly affect the fluid turbulence (an assumption that is increasingly valid for increasingly small $\tau_{p}$ ) and that they are dilute enough not to interact with each other. In the next section we introduce the KS model that we use. For this paper's purposes of demonstration it is enough to consider planar velocities and velocity fields, i.e., two components in a vertical plane. This does not mean to say, of course, that two- and three-dimensional turbulence are trivially interchangeable, as they of course have different dynamics. However, these dynamics are not incorporated in KS, which holds information only about the energy spectrum, incompressibility, and the time persistence of streamlines.

\section{TURBULENT LANGRANGIAN VELOCITIES}

We follow the approach of Turfus and Hunt [12], Sabelfeld [13], and Fung et al. [14] and generate on the computer an incompressible two-dimensional turbulentlike velocity field $\mathbf{u}(\mathbf{x}, t)$ that is identical to that of Vassilicos and Fung [15], i.e.,

$$
\mathbf{u}(\mathbf{x}, t)=\sum_{n=1}^{N_{k}}\left[\mathbf{A}_{n} \cos \left(\mathbf{k}_{n} \cdot \mathbf{x}+\omega_{n} t\right)+\mathbf{B}_{n} \sin \left(\mathbf{k}_{n} \cdot \mathbf{x}+\omega_{n} t\right)\right],
$$

where $N_{k}$ is the number of modes in the simulations and the Cartesian coordinates of $\mathbf{A}_{n}, \mathbf{B}_{n}$, and $\mathbf{k}_{n}$ are given by $\mathbf{A}_{n}$ $=A_{n}\left(\cos \phi_{n},-\sin \phi_{n}\right), \quad \mathbf{B}_{n}=B_{n}\left(-\cos \phi_{n}, \sin \phi_{n}\right), \quad$ and $\mathbf{k}_{n}$ $=k_{n}\left(\sin \phi_{n}, \cos \phi_{n}\right)$. The angles $\phi_{n}$ are random and uncorrelated with each other and the velocity field (4) is incompress- ible because $\mathbf{A}_{n} \cdot \mathbf{k}_{n}=\mathbf{B}_{n} \cdot \mathbf{k}_{n}=0$ for all $n$. The positive amplitudes $A_{n}$ and $B_{n}$ are chosen according to

$$
A_{n}^{2}=B_{n}^{2}=E\left(k_{n}\right) \Delta k_{n}
$$

where $E(k)$ is a prescribed Eulerian energy spectrum of the form

$$
E(k)=E_{0} L(k L)^{-5 / 3}
$$

in the range $2 \pi / L=k_{1} \leqslant k \leqslant k_{N_{k}}=2 \pi / \eta$ (where $L$ is the largest eddy length scale of the turbulence) and such that $E(k)$ $=0$ outside this range. $\Delta k_{n}=\left(k_{n+1}-k_{n-1}\right) / 2$ for $2 \leqslant N_{k}$ $\leqslant N_{k}-1, \Delta k_{1}=k_{2}-k_{1}$, and $\Delta k_{N_{k}}=k_{N_{k}}-k_{N_{k}-1}$. The distribution of wave numbers $k_{n}$ is geometric, i.e.,

$$
k_{n}=k_{1} \alpha^{n-1},
$$

where $\alpha$ is a dimensionless number which is a function of $L / \eta$ and $N_{k}$ because $k_{N_{k}}=2 \pi / \eta$. [Hence $\alpha=(L / \eta) 1 /\left(N_{k}\right.$ -1).] From $(1 / 2) u^{\prime 2}=\int E(k) d k$, it ensues that $u^{\prime 2} \approx 3 E_{0}$.

The frequencies $\omega_{n}$ in Eq. (1) determine the unsteadiness associated with wave mode $n$. We chose a model $[15,16]$ where the unsteadiness frequency $\omega_{n}$ is proportional to the eddy turnover time of wave mode $n$, i.e.,

$$
\omega_{n}=\lambda \sqrt{k_{n}^{3} E\left(k_{n}\right)},
$$

where $\lambda$ is a dimensionless constant.

The KS velocity fields simulated here are stationary in time, and the autocorrelation of Lagrangian velocities following fluid elements is $R^{L}(\tau)=\exp \left(-\tau / T_{L}\right)$ (see Fung and Vassilicos [16] Flohr and Vassilicos [17]). We find, in this paper's KS, that the integral length scale $\mathcal{L} \approx L / 3$. From numerical simulations of statistical ensembles of fluid element trajectories, we also find in this paper's KS that the Lagrangian integral time scale $T_{L} \approx 0.2 \mathcal{L} / E_{0}^{1 / 2}$.

It may be worth noting that in KS we prescribe a spatiotemporal structure for the flow via incompressibility and Eqs. (4)-(7). This spatiotemporal structure incorporates eddying and straining flow regions [14-16].

\section{SIMULATIONS AND RESULTS}

Denoting by $x$ and $y$ the horizontal and vertical coordinates, respectively $[y$ pointing upward, i.e., $\mathbf{g}=(0,-g)]$, the initial condition of our simulations consists of 4000 particles of relaxation time $\tau_{p 1}$ uniformly distributed along the horizontal line $y=0$ between $x=-L / 2$ and $L / 2$ ( $L$ is the largest eddy length scale of the turbulence) and 4000 particles of relaxation time $\tau_{p 2} \geqslant \tau_{p 1}$ also uniformly distributed along the same stretch of horizontal line (see Fig. 1). The two different types of particle are therefore perfectly well mixed with each other at time $t=0$. We also set $\mathbf{v}(0)=\mathbf{u}\left(\mathbf{x}_{p}(0), 0\right)$ [and we also tried starting particle velocities $\mathbf{v}(0)=\xi \mathbf{u}\left(\mathbf{x}_{p}(0), 0\right)$ with $\xi$ randomly distributed between -1 and 1 , without detecting any appreciable difference in our specific results]. The question is, do the different species of particles remain well mixed at later times?

In this paper we address this question by solving Eqs. (3a) 


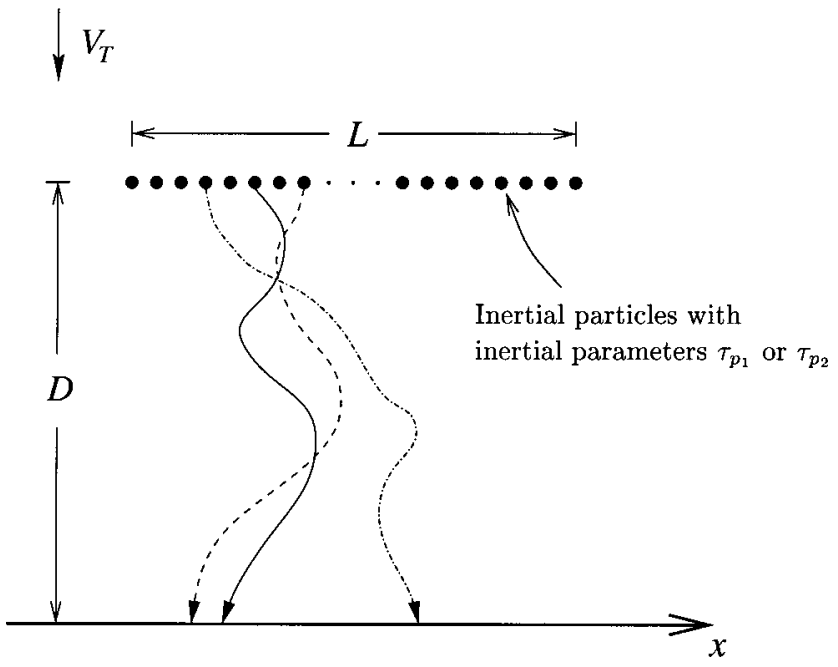

FIG. 1. Illustration of the numerical experiment and the motion of the particles under the influence of the flow field, the gravity, and their inertia.

and (3c) with the velocity field of the fluid turbulence specified by Eqs. (4)-(7). Values of the parameters $L, \eta, N_{k}$, and $\lambda$ used in this paper's KS are given in Table I. Values of the relaxation times $\tau_{p 1}$ and $\tau_{p 2}$ and of their ratio $r=\tau_{p 2} / \tau_{p 1}$ are given in Tables II-III. For comparison with the values of $\tau_{p 1}$ and $\tau_{p 2}$ in these tables, note that $T_{L} \approx 0.2$ in all the simulations here.

Inertial particle trajectories are integrated till they reach the horizontal line $y=-b L$ where their $x$ coordinates are recorded (in this paper we have experimented with $b=1$ and 2 and found no appreciable difference in our results, which have all been obtained for $\left.g \tau_{p 1}^{2} \leqslant L\right)$. These $x$ coordinates are used to derive two histograms $n_{1}(x)=n\left(\tau_{p 1}, x\right)$ and $n_{2}(x)$ $=n\left(\tau_{p 2}, x\right)$, which give the number of particles of relaxation times $\tau_{p 1}$ and $\tau_{p 2}$, respectively, that have crossed the horizontal line $y=-b L$ between $x$ and $x+\Delta x$ where $\Delta x$ is the histogram's bin size (see Fig. 2).

It is interesting to note from Figs. 2(a) and 2(b) that the histograms are irregular for both relaxation times $\tau_{p 1}$ and $\tau_{p 2}$. Hence the difference $n\left(\tau_{p 2}, x\right)-n\left(\tau_{p 1}, x\right)$ can be very significant [Fig. 2(c)]. We might therefore tentatively conclude that according to KS the turbulence can segregate the heavy and light particles. In what follows below, this tentative conclusion is corroborated and, more importantly perhaps, quantified by averaging over a large number of turbulent flow realizations. These averages also enable us to introduce a segregation length scale and discuss segregation properties as functions of the parameters of the problem.

TABLE I. Parameters used in kinematic simulation.

\begin{tabular}{cccccccc}
\hline \hline Case & $\tau_{p_{1}}$ & $\tau_{p_{2}}$ & $r$ & $\lambda$ & $L / \eta$ & $N_{k}$ & Falling distance \\
\hline $\mathrm{a}$ & 0.1 & 0.6 & 6 & 0.5 & 91 & 64 & $L$ \\
$\mathrm{~b}$ & 0.1 & 0.6 & 6 & 0.5 & 91 & 128 & $L$ \\
$\mathrm{c}$ & 0.1 & 0.6 & 6 & 0.5 & 273 & 256 & $L$ \\
$\mathrm{~d}$ & 0.1 & 0.6 & 6 & 0.5 & 91 & 64 & $2 L$ \\
\hline \hline
\end{tabular}

TABLE II. Ratio of relaxation times.

\begin{tabular}{lccr}
\hline \hline & \multicolumn{3}{c}{$\tau_{p_{2}} / \tau_{p_{1}}$} \\
Case 1 & Case 2 & Case 3 & $r$ \\
\hline $0.12 / 0.1$ & $0.144 / 0.12$ & $0.168 / 0.14$ & 1.2 \\
$0.14 / 0.1$ & $0.168 / 0.12$ & $0.196 / 0.14$ & 1.4 \\
$0.16 / 0.1$ & $0.192 / 0.12$ & $0.224 / 0.14$ & 1.6 \\
$0.18 / 0.1$ & $0.216 / 0.12$ & $0.252 / 0.14$ & 1.8 \\
$0.20 / 0.1$ & $0.240 / 0.12$ & $0.280 / 0.14$ & 2.0 \\
$0.40 / 0.1$ & $0.480 / 0.12$ & $0.560 / 0.14$ & 4.0 \\
$0.60 / 0.1$ & $0.720 / 0.12$ & $0.840 / 0.14$ & 6.0 \\
$0.80 / 0.1$ & $1.960 / 0.12$ & $1.120 / 0.14$ & 8.0 \\
$1.00 / 0.01$ & $1.200 / 0.12$ & $1.400 / 0.14$ & 10.0 \\
\hline \hline
\end{tabular}

The first and second moments of the difference between the two histograms are defined as follows:

$$
\begin{aligned}
\text { first moment: } & \left\langle\frac{1}{L} \int\left(n_{1}-n_{2}\right) d x\right\rangle=0, \\
\text { second moment: } & \left\langle\frac{1}{L} \int\left(n_{1}-n_{2}\right)^{2} d x\right\rangle=M_{2},
\end{aligned}
$$

where the angular brackets signify an average over 150 turbulent flow realizations. Note that the first moment is always equal to zero.

In all our runs, the smallest time scale of the turbulence is smaller than $\tau_{p 1}$ and $\tau_{p 2}$ [which is consistent with our requirements that $\rho_{p} \gg \rho_{f}$ and $a \ll \eta$, provided that $\rho_{p} / \rho_{f}$ $\left.\gg(\eta / a)^{2}\right]$, and in KS this smallest time scale is $\tau_{\eta}$ $\sim 1 / \sqrt{k_{N_{k}}^{3} E\left(k_{N_{k}}\right)}$. Hence, we may assume that $M_{2}$ is independent of this smallest time scale, and the parametric dependence of $M_{2}$ for small enough bin size is given by

$$
\begin{aligned}
M_{2} & =M_{2}\left(u^{\prime}, T_{L}, \tau_{p 1}, \tau_{p 2}, g\right) \\
& =(\Delta x / L)^{2} m_{2}\left(r, \tau_{p 1} / T_{L}, g T_{L} / u^{\prime}\right),
\end{aligned}
$$

where $m_{2}$ is a dimensionless function. In Fig. 3 we plot the dependence of $M_{2}$ on the two dimensionless parameters $r$ ( $r=\tau_{p 2} / \tau_{p 1}$ is larger than 1 by definition) and $\tau_{p 1} / T_{L}$. Note that the factor $10^{4}$ multiplying $M_{2}$ in these figures is effectively the inverse of $(\Delta x / L)^{2}$ as $\Delta x / L=O\left(10^{-2}\right)$ since $\Delta x$ $\approx \eta$ in our runs. Two regimes should readily be distinguished depending on whether $\tau_{p 1}$ is larger or smaller than $T_{L}$ : when $T_{L} \ll \tau_{p 1}$ we should expect $M_{2} \approx 0$ because no segregation can be expected when the relaxation time of both types of particles is much larger than the Lagrangian correlation time

TABLE III. Values of relaxation times.

\begin{tabular}{lll}
\hline \hline$\tau_{p_{1}}$ & $\tau_{p_{2}}$ & $r$ \\
\hline 0.2 & 1.2 & 6 \\
0.3 & 1.8 & 6 \\
0.4 & 2.4 & 6 \\
0.5 & 3.0 & 6 \\
\hline \hline
\end{tabular}



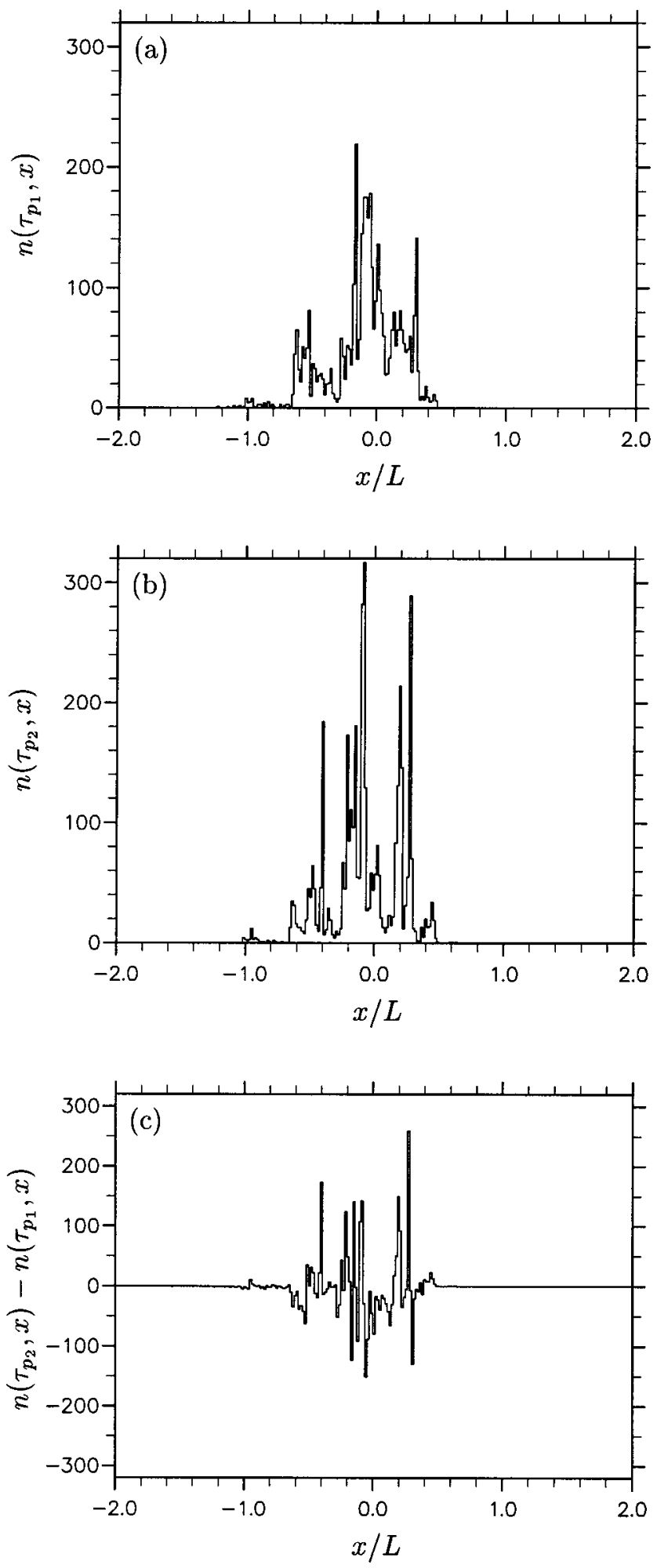

FIG. 2. One typical case of the histograms $n\left(\tau_{p_{i}}, x\right) \quad(i=1,2)$ along the horizontal line $y=-L$ in the KS case. Here $T_{L}=0.2$, $u^{\prime} \approx 1$, and $g=10$. Also, $2 \pi / L=1.1,2 \pi / \eta=100, N_{k}=64$, and the unsteadiness parameter $\lambda=0.5$. (a) $\tau_{p 1}=0.1$, (b) $\tau_{p 2}=0.4$, and (c) the difference $n\left(\tau_{p 2}, x\right)-n\left(\tau_{p 1}, x\right)$.

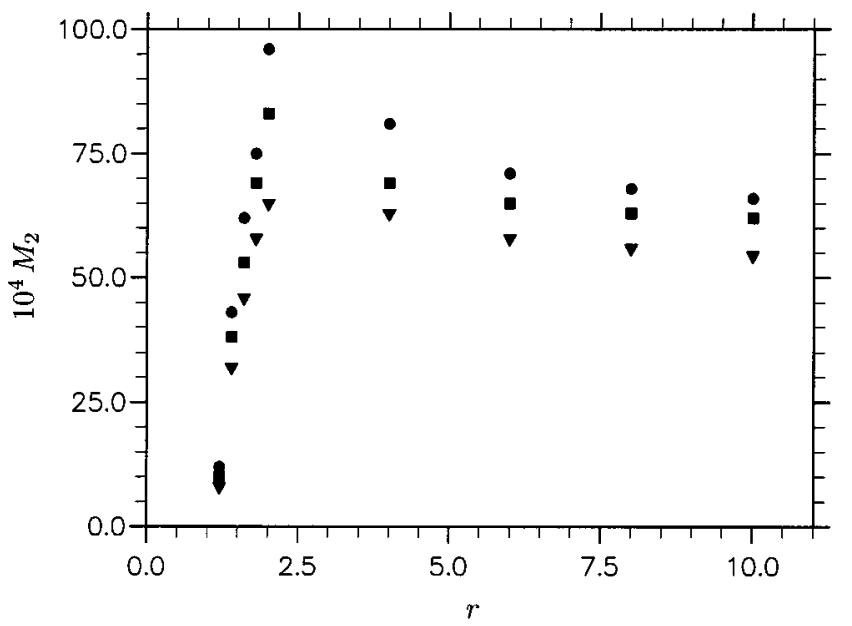

FIG. 3. $M_{2}$ as a function of $r$ for different values of $\tau_{p 1} / T_{L}$ obtained with KS. These different values are listed in Table II. The parameters determining the KS are the same as in Fig. 2. $\bullet$, case 1 with $\tau_{p 1}=0.1, \mathbf{\square}$, case 2 with $\tau_{p 1}=0.12$, and $\boldsymbol{\nabla}$, case 3 with $\tau_{p 1}$ $=0.14$.

of the turbulence; and when $\tau_{p 1} \leqslant T_{L}$ we should expect $M_{2}$ to be an increasing function of $r$ in the range $1 \leqslant r \leqslant T_{L} / \tau_{p 1}$ because of the increasing difference in particle relaxation times, but asymptotically constant in the limit $r \gg T_{L} / \tau_{p 1}$ because in that limit the relaxation time of one of the two particles is larger than $T_{L}$. These qualitative expectations are confirmed in Fig. 3, even though the case $M_{2} \approx 0$ is not absolutely evident from the data; note, however, that for $r$ $\gg T_{L} / \tau_{p 1}, M_{2}$ decreases with increasing $\tau_{p 1} / T_{L}$, presumably reaching zero when $\tau_{p 1} / T_{L} \gg 1$. We also found these results not to depend significantly on $g$, at least for the few values of $g$ that we tried $\left(g T_{L} / u^{\prime}=2,3,4\right)$. Note also that, for $r \gg T_{L} / \tau_{p 1}, M_{2}$ decreases with increasing $\tau_{p 1} / T_{L}$, presumably reaching zero when $\tau_{p 1} / T_{L} \gg 1$.

In order to define a characteristic length scale of segregation, $l_{c}$, we Fourier transform $n\left(\tau_{p 1}, x\right)-n\left(\tau_{p 2}, x\right)$ and calculate the power spectrum $\Phi(k)$ of $n\left(\tau_{p 1}, x\right)-n\left(\tau_{p 2}, x\right)$ [with an average over 150 turbulent flow realizations so that $\left.\int \Phi(k) d k=M_{2}\right]$. If a well-defined length scale characterizing segregation does exist, this power spectrum should be clearly peaked at a wave number $2 \pi / l_{c}$, which should be significantly larger than $2 \pi / L_{D}$, where $L_{D}$ is the length of the total extent of the horizontal line $y=-b L$ that the particles have crossed $\left(L_{D}\right.$ is significantly larger than $\left.L\right)$. In Figs. 4 and 5 we plot $\Phi(k)$ in the range $2 \pi / L_{D} \leqslant k$ $\leqslant 2 \pi / \Delta x$ for a number of different parameters (see Tables II and III). The first and broad conclusion that can be drawn from these figures is that a well-defined characteristic length scale of segregation $l_{c}$ exists that is clearly smaller than $L_{D}$. (We find no significant variability in these spectra for $g T_{L} / u^{\prime}=2,3,4$ except a slight one in the value of $l_{c}$.) Also, the power spectrum $\Phi(k)$ appears power-law shaped in some cases.

A finer analysis of these results reveals the parametric variability of $l_{c}$. Because we consider values of $\tau_{p 1}$ and $\tau_{p 2}$ larger than $\tau_{\eta}, l_{c}$ may be expected to be a function of $u^{\prime}$, $T_{L}, g, \tau_{p 1}$, and $\tau_{p 2}$ only, so that by dimensional analysis

$$
l_{c} / L=f\left(r, \tau_{p 1} / T_{L}, g T_{L} / u^{\prime}\right)
$$



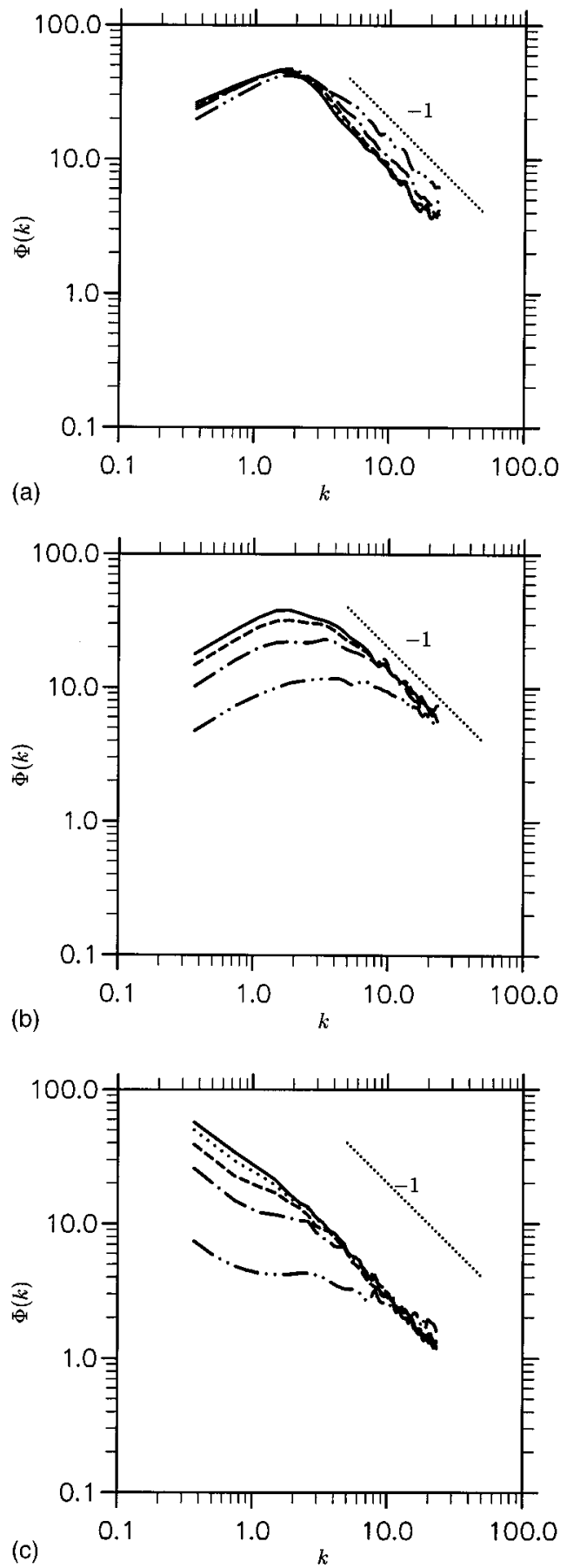

FIG. 4. KS power spectra $\Phi(k)$ vs $k$ for different inertial ratios $r=\tau_{p 2} / \tau_{p 1}$ with fixed $\tau_{p 1}=0.1$. (a) $\tau_{p 1}<T_{L} \leqslant \tau_{p 2} . T_{L}=0.2$ and $\tau_{p 2}$ varies from 0.2 to 1.0 so that $r$ varies from 2 to 10 . This figure corresponds to case 1 in Table II. The parameters determining the KS are the same as in Fig. 2. $r=2$ (dot-dot-dashed), 4 (dot-dashed), 6 (dashed), 8 (dotted), and 10 (solid). (b) $\tau_{p 1}<\tau_{p 2}<T_{L} \cdot T_{L}=0.2$ and $\tau_{p 2}$ varies from 0.12 to 0.18 so that $r$ varies from 1.2 to 1.8 . This figure corresponds to case 1 in Table II. The parameters determining the KS are the same as in Fig. 2. $r=1.2$ (dot-dot-dashed), 1.4 (dot-dashed), 1.6 (dashed), and 1.8 (solid). (c) The same as (a) but with $g=1.0 . r=2$ (dot-dot-dashed), 4 (dot-dashed), 6 (dashed), 8 (dotted), and 10 (solid).

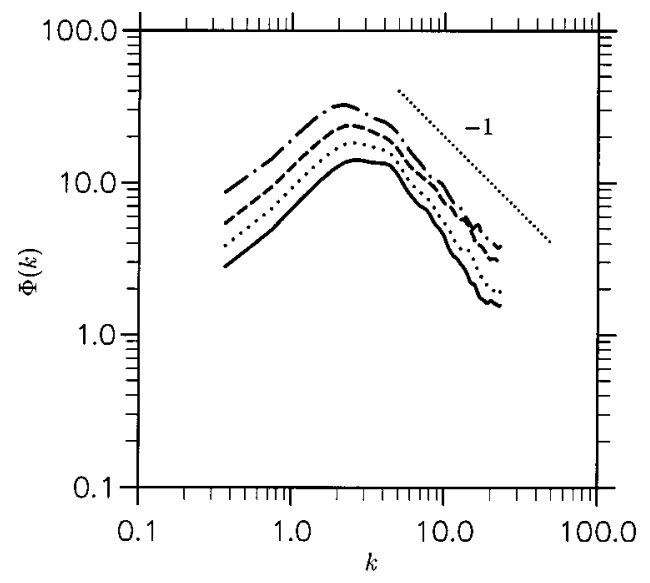

FIG. 5. KS power spectra $\Phi(k)$ vs $k$ for fixed inertial ratio $r$ $=6$ and varying $\tau_{p_{1}}$ such that $T_{L} \leqslant \tau_{p 1}<\tau_{p 2} . T_{L}=0.2$ and $\tau_{p 1}$ with fixed inertial ratio $r$ and $\tau_{p 1}$ varies from 0.2 to 0.5 . This figure corresponds to Table III. The parameters determining the KS are the same as in Fig. 2. $\tau_{p 1}=0.2$ (dot-dashed), 0.3 (dashed), 0.4 (dotted), and 0.5 (solid)

where $f$ is a dimensionless function. Again we distinguish between the following two regimes: $T_{L} \ll \tau_{p 1}$, in which case we expect $l_{c} \approx 0$; and $\tau_{p 1}<T_{L}$, in which case we should expect $l_{c}$ to be an increasing function of $r$ in the range 1 $\leqslant r \leqslant T_{L} / \tau_{p 1}$ but asymptotically constant in the limit $r$ $\gg T_{L} / \tau_{p 1}$. This is indeed what is observed (see Fig. 6).

Power spectra $\Phi(k)$ for cases where $\tau_{p 1}<T_{L} \leqslant \tau_{p 2}$ are given in Fig. 4(a), where it may be observed that $\Phi(k)$ $\sim k^{-1}$ for $k>2 \pi / l_{c}$ and $r \gg 1$. Power spectra $\Phi(k)$ for cases where $\tau_{p 1}<\tau_{p 2}<T_{L}$ are presented in Fig. 4(b), and, finally, power spectra $\Phi(k)$ for cases where $T_{L} \leqslant \tau_{p 1}<\tau_{p 2}$ are given in Fig. 5, where $\Phi(k)$ may be observed to decay faster than $k^{-1}$ for $k>2 \pi / l_{c}$ and in fact faster for larger values of $\tau_{p 1}$ and $\tau_{p 2}$.

A simple dimensional argument leading to $\Phi(k) \sim k^{-1}$ for $k>2 \pi / l_{c}$ in the case $\tau_{p 1}<T_{L} \ll \tau_{p 2}$ and $g \tau_{p 1}^{1 / 2} L^{1 / 2} / u^{\prime 3 / 2}$

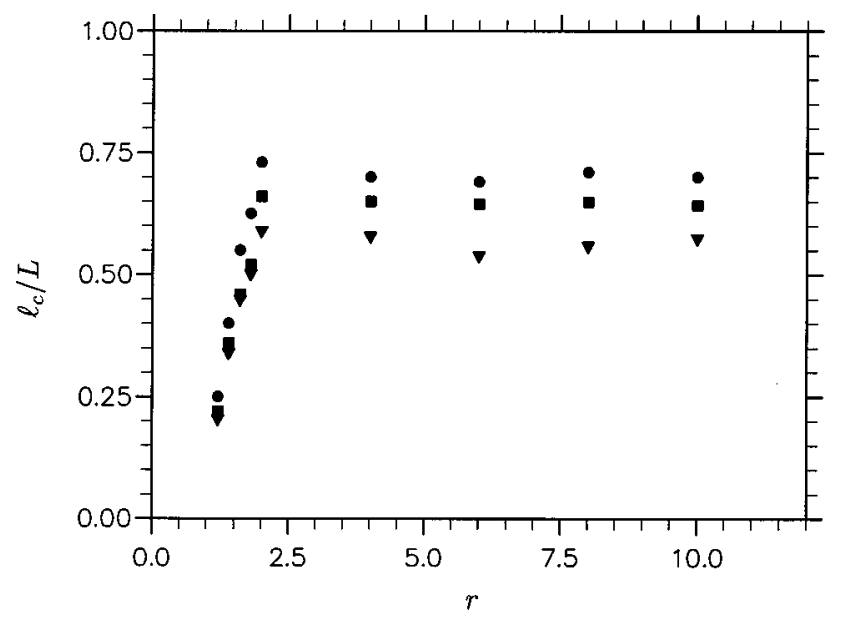

FIG. 6. Characteristic length scale of segregation, $l_{c}$, as a function of $r$ for different values of $\tau_{p 1} / T_{L}$ obtained with KS. These different values are listed in Table II. The parameters determining the KS are the same as in Fig. 2. $\bigcirc$, case 1 with $\tau_{p 1}=0.1, \boldsymbol{\square}$, case 2 with $\tau_{p 1}=0.12$, and $\boldsymbol{\nabla}$, case 3 with $\tau_{p 1}=0.14$. 


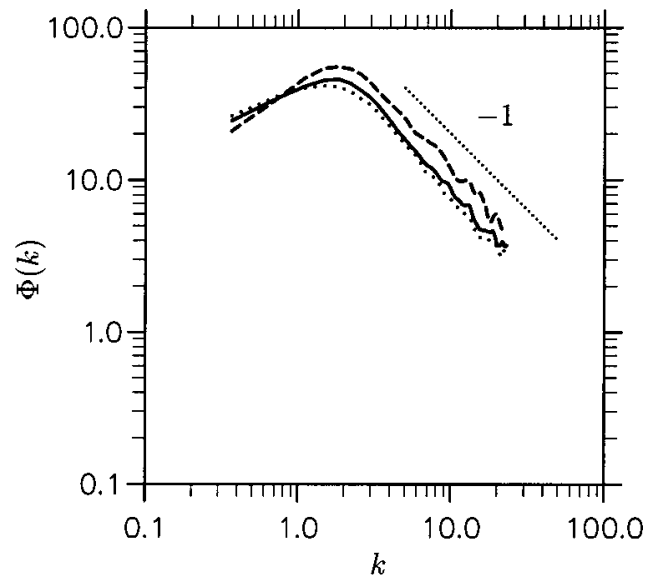

FIG. 7. KS power spectra $\Phi(k)$ vs $k$ with $2 \pi / L=1.1,2 \pi / \eta$ $=100, N_{k}=64, T_{L}=0.2, g=10, u^{\prime} \approx 1$, fixed inertial ratio $r=6$, and fixed $\tau_{p 1}=0.1$, but with different unsteadiness parameter $\lambda=0.3$ (dashed), 0.5 (solid), and 0.7 (dotted).

$\leqslant O$ (1) can be developed as follows. The power spectrum $\Phi(k)$ cannot depend on $\tau_{\eta}$ for reasons already mentioned, and cannot depend on $\tau_{p 2}$ either in the limit where $T_{L}$ $\ll \tau_{p 2}$. Furthermore, in the range $k>2 \pi / l_{c}$, small-scale segregation and $\Phi(k)$ cannot be expected to depend on the large-scale quantities $L$ and $u^{\prime}$. Hence, by dimensional constraints,

$$
\Phi(k)=\Phi\left(k, g, \tau_{p 1}\right)=k^{-1} \phi\left(k g \tau_{p 1}^{2}\right)
$$

where $\phi$ is a dimensionless function.

The gravitational acceleration $g$ cannot by itself segregate particles of different relaxation times. However, $g$ can indirectly influence this segregation by influencing the time taken for particles to fall through the eddies. Nevertheless, when the characteristic vertical distance $g \tau_{p 1}^{2}$ that a particle would travel by gravitation alone in the course of a relaxation time $\tau_{p 1}$ is much smaller than the size of the eddy, then we might expect $g$ not to influence the time taken by the particle to fall through this eddy and therefore Eq. (10) not to depend on $g$. The smallest eddy size that can influence segregation is not $\eta$ but instead the size of the eddy with characteristic time equal to $\tau_{p 1}$. The characteristic time for an eddy of size $k^{-1}$ is $1 / \sqrt{k^{3} E(k)}$ (see the KS description in the previous section). Hence, the smallest eddy size that can cause segregation is $L\left(u^{\prime} \tau_{p 1} / L\right)^{3 / 2}$. The condition for Eq. (10) not to depend on $g$ is therefore $g \tau_{p 1}^{2} \leqslant L\left(u^{\prime} \tau_{p 1} / L\right)^{3 / 2}$, i.e., approximately $g \tau_{p 1}^{1 / 2} L^{1 / 2} / u^{\prime 3 / 2} \leqslant O(1)$. Dimensional requirements imply that in this regime the spectrum scales like

$$
\Phi(k) \sim k^{-1},
$$

in agreement with the power-law spectra reported in Fig. 4(a) and also in Fig. 4(c), where the tendency for a collapse as $r$ increases is perhaps clearer because $g \tau_{p 1}^{1 / 2} L^{1 / 2} / u^{\prime 3 / 2}$ is smaller than in Fig. 4(a).

Finally, we also report Figs. 7 and 8 where evidence is presented showing that $\Phi(k)$ is insensitive to the unsteadi-

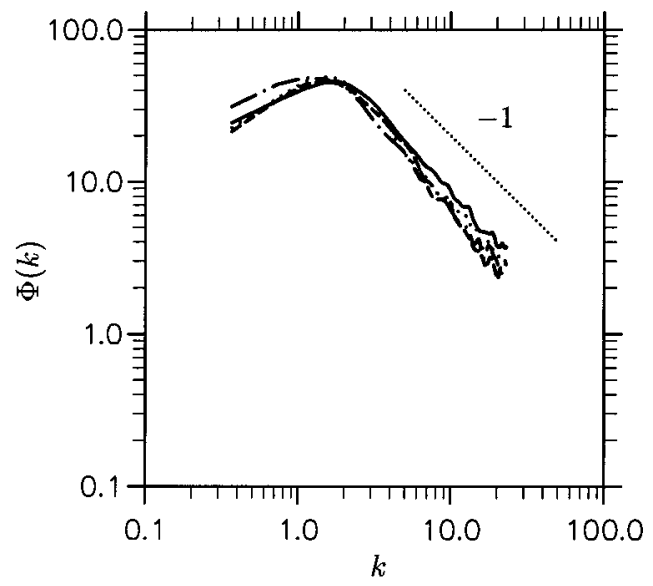

FIG. 8. KS power spectra $\Phi(k)$ vs $k$ with $2 \pi / L=1.1, T_{L}$ $=0.2, g=10, u^{\prime} \approx 1, \lambda=0.5, r=6$, and $\tau_{p 1}=0.1 . N_{k}=64, L / \eta$ $=91$, falling distance $D=-L$ (solid); $N_{k}=128, L / \eta=91$, falling distance $D=-L$ (dotted); $N_{k}=256, L / \eta=273$, falling distance $D$ $=-L$ (dashed); and $N_{k}=64, L / \eta=91$, falling distance $D=-2 L$ (dot-dashed).

ness parameter $\lambda$ in the range between 0.3 and 0.8 and also independent of $\eta$ (and therefore $\tau_{\eta}$ as assumed in our arguments above).

\section{CONCLUSION}

We have introduced a segregation power spectrum $\Phi(k)$ and a segregation length scale $l_{c}$ and demonstrated that $l_{c}$ is well defined in a kinematic simulation of particle dispersion. For particles of very different relaxation times, and for wave numbers larger than $l_{c}^{-1}$, it is found that $\Phi(k) \sim k^{-1}$ provided that $\tau_{\eta} \leqslant \tau_{p 1}<T_{L} \ll \tau_{p 2}$ and $g \tau_{p 1}^{1 / 2} L^{1 / 2} / u^{\prime 3 / 2} \leqslant O(1)$. The present study also supports the view that spatiotemporal flow structure can cause particles of different inertias to segregate in a turbulent flow. This conclusion is consistent with the results of Maxey [1], Squires and Eaton [2], Wang and Maxey [3], and Fung [4], who have shown that the flow structure of the small-scale turbulence influences the concentration fields of dense particles. The effect of intense and persistent local vortical structure can quickly modify the particle concentration field near these local regions. Particles tend to accumulate in regions of high flow strain rate or low flow vorticity because of an inertial bias. This preferential accumulation has been confirmed by the results from full direct numerical simulations in homogeneous turbulence by Squires and Eaton [2] and Wang and Maxey [3], and by kinematics simulations in homogeneous turbulence by Maxey [1] and Fung [4]. We hope the present study indicates that the structural view of turbulent flows can contribute to the development of multiphase flow modeling, in addition to the more commonly used statistical view.

\section{ACKNOWLEDGMENTS}

Support from the Hong Kong Research Grant Council, China (Project Nos. HKUST6121/00P and HKUST6012/ 03P) and from the Royal Society is gratefully acknowledged. 
[1] M. R. Maxey, J. Fluid Mech. 174, 441 (1987).

[2] K. D. Squires and J. K. Eaton, Phys. Fluids 3, 1169 (1991).

[3] L. P. Wang and R. M. Maxey, J. Fluid Mech. 256, 27 (1993).

[4] J. C. H. Fung, J. Geophys. Res., [Oceans] 98(C11), 20287 (1993)

[5] A. M. Ahmed and S. Elghobashi, Phys. Fluids 13, 3346 (2001).

[6] D. J. Vojir and E. E. Michaelides, Int. J. Multiphase Flow 20, 547 (1994).

[7] J. C. R. Hunt, R. J. Perkins, and J. C. H. Fung, Appl. Mech. Rev. 47, S49 (1994).

[8] R. W. Mei, J. Fluid Mech. 270, 133 (1994).

[9] M. W. Reeks, J. Fluid Mech. 97, 569 (1980).

[10] L. P. Wang and D. E. Stock, ASME J. Fluids Eng. 114, 100
(1992).

[11] J. C. H. Fung, J. Geophys. Res., [Oceans] 103(C12), 27905 (1998).

[12] C. Turfus and J. C. R. Hunt, in Advances in Turbulence, edited by G. Comte-Bellot and J. Mathieu (Springer-Verlag, Berlin, 1986).

[13] K. Sabelfeld, Monte Carlo Methods (Springer-Verlag, Berlin, 1991).

[14] J. C. H. Fung, J. C. R. Hunt, N. A. Malik, and R. J. Perkins, J. Fluid Mech. 236, 281 (1992).

[15] J. C. Vassilicos and J. C. H. Fung, Phys. Fluids 7, 1970 (1995).

[16] J. C. H. Fung and J. C. Vassilicos, Phys. Rev. E 57, 1677 (1998).

[17] P. Flohr and J. C. Vassilicos, J. Fluid Mech. 407, 351 (2000). 